\title{
ACHIEVEMENT OF ZVT AND COMPUTATION OF ITS TIME OF OCCURRENCE FOR POWER CONVERTERS
}

\author{
V.F. Montagner ${ }^{1}$, H.L. Hey ${ }^{1}$, M.L. Martins ${ }^{2}$, J.L. Russi ${ }^{3}$ \\ ${ }^{1}$ Federal University of Santa Maria, GEPOC, Santa Maria, RS, Brazil. \\ ${ }^{2}$ Federal University of Technology - Paraná, UTFPR, Campus Pato Branco, PR, Brazil . \\ ${ }^{3}$ Federal University of Pampa - UNIPAMPA, Campus Alegrete, RS, Brazil. \\ \{vfmontagner; heliohey; mariolsm; jrussi\}@ gmail.com
}

\begin{abstract}
This paper provides a mathematical expression to determine the occurrence of soft-switching for a general topology of ZVT converters with auxiliary resonant voltage source. This expression is used to help the designer in choosing appropriately the values of inductance and capacitance for the auxiliary resonant branch ensuring ZVT. The main advantage of the proposed methodology is to point out suitable sets of circuit parameters without relying only on time consuming numerical simulations of the entire converter. Additionally, numerical methods to compute the time of occurrence of ZVT for these power converters are provided. The proposed methods are given by second order approximations of a nonlinear function which describes the voltage on the ZVT snubber capacitor during the zero-voltage resonant stage. The results can be seen as applications of second order Taylor series and of quadratic interpolation. However, differently of simply finding the root of a given nonlinear equation, the conditions given here provide analytical expressions to compute the time of occurrence of $\mathrm{ZVT}$ based on the converter parameters. Then, the proposed conditions become useful for analysis and design, allowing evaluating operation sequence, total commutation time, resistive losses and other important parameters to describe the converter performance.
\end{abstract}

Keywords - Design Guidelines, Mathematical Optimization, Soft-Switching, ZVT.

\section{INTRODUCTION}

The continuous search for compactness and high performance of power electronics converters always push the switching frequency of the semiconductors to high values. To overcome the losses and electromagnetic interference problems associated with such high frequencies, softswitching approaches have been extensively used. In the last decades, several soft-switching topologies have been proposed in power electronics literature [1-12]. Among the soft-switching techniques utilized so far are the Zero Voltage Transition, ZVT, converters with a resonant voltage source [7-12]. This class of ZVT converters operates in such a way that an active resonant snubber, comprised by a coupled of capacitors and an inductor, shapes the voltage and current waveforms of the switching device before its commutation, alleviating the switching energy losses. Hence, the choice of

Manuscript received on 04/19/2010. Accepted for publication on 07/10/2010 by recommendation of the Editor João Onofre P. Pinto. the active resonant snubber parameters are of prime concern to ensure the best performance of the soft-switching technique and thus, it still deserves investigation on how to choose circuit parameters (specially the inductance and capacitance of the auxiliary branch and occurrence of ZVT) based on performance indices that rely on analytical conditions (i.e. mathematical expressions) instead of on only computer simulation. Although simulation is recognized as a fundamental tool to help the circuit designer, the existence of analytical tests ensuring properties as ZVT and other important performance parameters can contribute to increase the reliability of the results and to reduce the time spent in the design.

The first contribution of this paper is to provide an analytical condition to decide whether a resonant stage with switch voltage given by

$$
v(t)=A+B t+C \sin \left(\omega_{e} t\right)+D \cos \left(\omega_{e} t\right)
$$

achieves ZVT or not. The proposed condition avoids numerical simulation to detect a real positive root for the above nonlinear function. Instead of this, it uses mathematical optimization to check the existence of ZVT by means of an analytical condition [13,14]. An immediate use of this condition is to rapidly decide for which values of resonant inductance and capacitance, in a set defined by the circuit designer, ZVT is ensured.

Then, some mathematical methods are provided in order to compute, by means of analytical approximations, the time of occurrence of ZVT in a resonant stage with switch voltage given by the former expression.

In other words, it aims on the computation of the root of $v(t)$ in the general case, where the parameters of the equation are not known. The motivation for this work is the importance of having mathematical conditions that, based on the knowledge of $A, B, C, D$ and $\omega_{e}$, and without relying only on computer simulation, provide the time at which ZVS takes place. Such conditions allow the systematic calculation of important features such as operation sequence and quantities as the total commutation time, the RMS value of currents that are important for circuit operation and that are related with resistive losses, to the loss of duty ratio, etc.

The above function is nonlinear and its root is nontrivial to be obtained analytically. It will be shown next that a simple second order Taylor series, centered at appropriately chosen points, and a quadratic interpolation provide the value of the root of this function with good precision [14].

The analytical approach proposed here to calculate the root of $v(t)$ is based on two simple steps: first, before searching the root of $v(t)$, it must be assured that the root exists; second, instead of searching the root by numerical 
computation in a case by case basis, it can be used mathematical expressions that relate the value of an acceptable approximation for the root with parameters $A, B$, $C, D$ and $\omega_{e}$. The advantages of this approach is saving time in searching the root when it does not exist and not being a case by case solution, but being a systematic solution for the problem. The usefulness and the efficiency of the proposed conditions are illustrated by means of numerical examples in the paper.

This paper is organized as follows: in Section II the operation sequences of the concerning converters is described; section III provides the analytical condition to decide if ZVT occurs or not and an example of application of this condition; Section IV describes three methods to obtain the center point of the Taylor series which describes the behavior of $v(t)$; Section $\mathrm{V}$ shows some examples for the methods proposed and shows how to determine two operation parameters that can be obtained by the approaches shown in Section III.

\section{STRUCTURE OF A ZVT TOPOLOGY}

Despite the large amount of known ZVT converters, all of them share the same basic structure, which is illustrated in Figure 1(a). As shown in this figure, there are capacitors placed in parallel to the switches of the converter pole. These capacitances aim to slow down the $d v / d t$ rates when a switch is turned off. As they mitigate the turn-off losses by decreasing the overlap between the voltage and current waveforms through a switch, they also would cause large turn-on losses when the switch is turned on since the energy stored on the plates of the capacitor would be dissipated in the switch. Such drawback is avoided by discharging the capacitance before the turn-on of a switch, what is accomplished by a circuitry that works similarly as a current source, injecting or drawing current from the pole. The least amount of current that must be removed from the pole is given by (2).

$$
\int_{\left.t\right|_{i a=I s}}^{t f}\left(i_{C S}(t)-I_{S}\right) d t=\int_{\left.t\right|_{i a=I s}}^{t f}\left(i_{a}(t)-I_{S}\right) d t=C_{S} V_{Z Y},
$$

where $C_{S}=C_{S 1}+C_{S 2}$.

Equation (2) shows that the current drawn/injected in the pole can be of an arbitrary shape as long as it is larger than $I_{S}$ and it remains larger enough time to discharge $C_{S}$, the resulting capacitance.

In practical circuits the current source $I_{a}$ is implemented by an inductor whose current is controlled by the voltage applied across it, Figure 1(b). As a result, the inductor current is directly a function of the way as a voltage source connected to the inductor branch behaves during the switches turn-on commutation interval.

One of the ways of implementing the voltage source $E_{a}$ from Figure 1(b) is by means of a capacitor. Since the voltage of a capacitor is not a constant value, it must be assured that it presents the adequate value (or range of values) during the commutation process, what can be obtained by clamping circuits to avoid the capacitor voltage boosting or/and by the connection of terminal $a$ to some terminal of the converter, Figure 1(c). The occurrence or not of the clamping results in three different operation sequences, which will be commented further on. The voltage applied on terminal $a$ in relation to terminal $y$ will be called $V_{W}$ and is a function of the topology under analysis. Its value is $V_{o}$ for [8] and zero for [7], [9-12].

Consider that $I_{S}$ current flows into the pole and flows entirely through the body diode of switch $\mathrm{S}_{2}$. Consider also $C_{S}$ as the equivalent capacitance from $C_{S 1}$ and $C_{S 2}$. The basic operation of the converter for the turn-on and turn-off of $S_{1}$ can be summarized into the stages shown in Figure 2.

(i) Initially the entire current $I_{S}$ flows through the antiparallel diode of switch $S_{2}\left(D_{S 2}\right)$. (ii) Then, to commutate from $D_{\mathrm{S} 2}$ to $S_{1}$, the current through the Auxiliary Branch, $\mathrm{AB}$, starts to increase resonantly until it reaches the $I_{S}$ current value. (iii-a) When this happens, capacitor $C_{S 1}$ joins the resonating process being completely discharged. (iv-a) The $\mathrm{AB}$ continues to resonate until the current through it becomes null, (v) inverts its sense and resonates until $C_{r}$ reaches its initial conditions. (vi) Then, the entire $I_{S}$ current flows through switch $\mathrm{S}_{1}$. (vii) When $\mathrm{S}_{1}$ is turned off, $C_{S 1}$ is charged due to $I_{S}$ up to the $V_{Z Y}$ voltage.

There are three possible operations sequences. In the first sequence, which was detailed above, the clamping voltage of

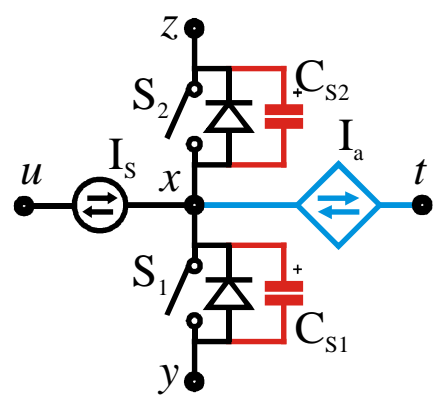

(a)

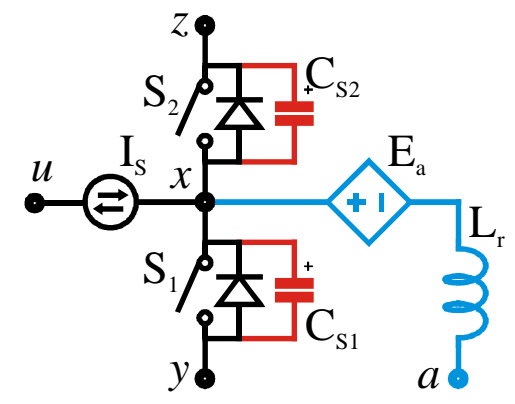

(b)

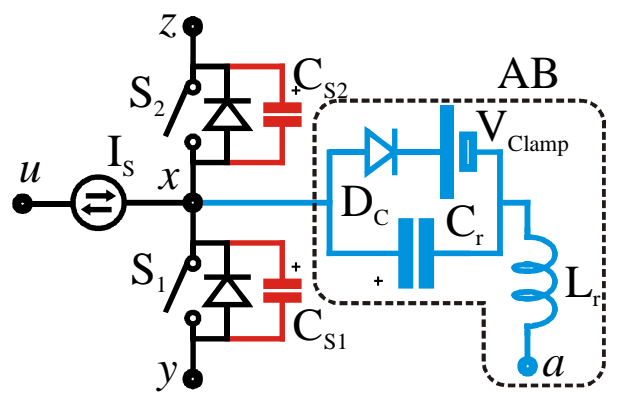

(c)

Fig. 1. Diagrams for ZVT structures. (a) General structure; (b) ZVT structure with practical auxiliary current source; (c) ZVT topology with a capacitor as the auxiliary voltage source. 


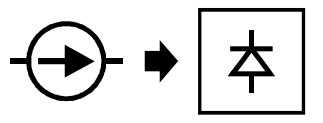

(i)

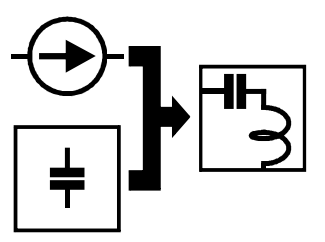

(iii-a)

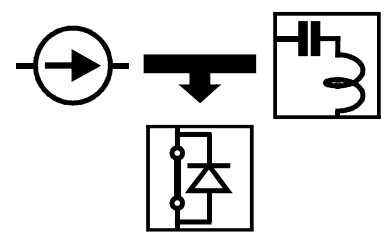

(iv-a)

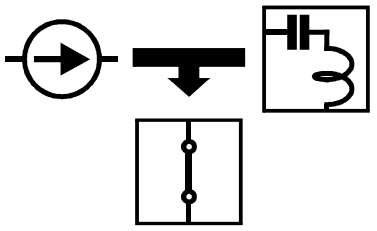

(v)

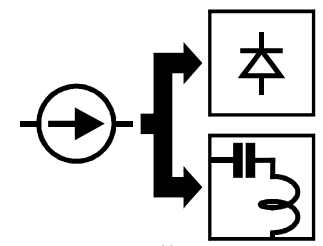

(ii)

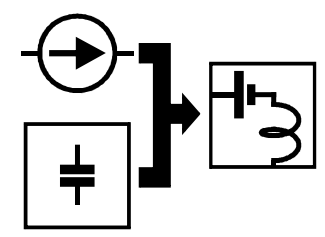

(iii-b)

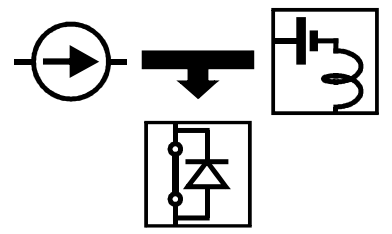

(iv-b)

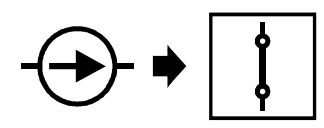

(vi)

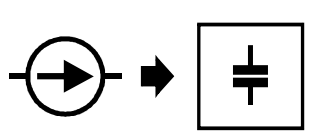

(vii)
Fig. 2. Diagrams for the ZVT operation circuit modes.

capacitor $C_{r}$ is not reached; in sequence two, it is reached during stage (iii-a). As a result, (iii-b) the energy from $C_{r}$ stops resonating and (iv-b) $L_{r}$ discharges linearly. With $L_{r}$ discharged, the operation resumes from (v). The last possibility is that the clamping voltage of $C_{r}$ is reached during stage (iv-a). In this case (iv-b), $L_{r}$ discharges linearly and the operation also resumes from stage (v).

As commented above:

Sequence 1: i, ii, iii-a, iv-a, v, vi and vii;

Sequence 2: i, ii, iii-a, iii-b, iv-b, v, vi and vii; and

Sequence 3: i, ii, iii-a, iv-a, iv-b, v, vi and vii.

\section{ANALYTICAL CONDITION FOR ZVT}

Assume that the current through $\mathrm{AB}$ reaches $I_{S}$ at the end of stage (ii), that is, there exists a real positive solution for function (1). Then, the voltage through $S_{1}$ is given by (1).

The parameters $A, B, C$ and $D$ in (1) depend on the parameters of the circuit and on the final values of state variables in stage (ii), being written as:

$$
\begin{gathered}
A=\left(\left(V_{C_{r}}\left(t_{2}\right)+V_{W}\right) C_{r}+V_{o} C_{S}\right) /\left(C_{r}+C_{S}\right), \\
B=I /\left(C_{r}+C_{S}\right), \\
C=-I /\left(\left(C_{r}+C_{S}\right) \omega_{e}\right)
\end{gathered}
$$

$$
D=C_{r}\left(V_{o}-V_{W}-V_{C_{r}}\left(t_{2}\right)\right) /\left(C_{r}+C_{S}\right),
$$

where $V_{C r}\left(t_{2}\right)$ is given by

$$
\begin{gathered}
V_{C_{r}}\left(t_{2}\right)=V_{o}-V_{W}+\left(V_{C_{r}}\left(t_{0}\right)+V_{W}-V_{o}\right) \cos \left(\omega_{r} t_{2}\right) \\
t_{2}=\arcsin \left(I Z_{r} /\left(V_{o}-V_{C_{r}}\left(t_{0}\right)-V_{W}\right)\right) / \omega_{r},
\end{gathered}
$$

and is equivalent to

$$
V_{C_{r}}\left(t_{2}\right)=V_{o}-V_{W}-\sqrt{\left(V_{C_{r}}\left(t_{0}\right)+V_{W}-V_{o}\right)^{2}-\left(I Z_{r}\right)^{2}}
$$

with $V_{C r}\left(t_{0}\right)<0, t_{2}$ from equation (8) and

$$
\begin{gathered}
\omega_{e}=\sqrt{\frac{C_{r}+C_{S}}{L_{r} C_{r} C_{S}}}, \\
Z_{r}=\sqrt{L_{r} / C_{r}}
\end{gathered}
$$

and

$$
\omega_{r}=1 / \sqrt{L_{r} C_{r}} .
$$

The knowledge of the signal of some of these terms will be useful furthermore. By inspection of the previous expressions, it follows that $v(0)=A+D=V_{o}$ and thus $v(0)>0$. Moreover, $B>0, C<0$ and $D>0$. Particularly, $D>0$ holds for two situations. First, for $V_{W}=V_{o}$, one has

$$
D=\frac{C_{r}\left(-V_{C_{r}}\left(t_{2}\right)\right)}{C_{r}+C_{S}}=\frac{C_{r}\left(-V_{C_{r}}\left(t_{0}\right) \cos \left(\omega_{r} t_{2}\right)\right)}{C_{r}+C_{S}} .
$$

Since, from the existence of solution of (1), one has $\left.\sin \left(\omega_{r} t_{2}\right)=\frac{I Z_{r}}{-V_{C_{r}}\left(t_{0}\right)} \in\right] 0,1\left[\right.$ then $\left.\omega_{r} t_{2} \in\right] 0, \pi / 2[$, leading to $\cos \left(\omega_{r} t_{2}\right)>0$ and hence $D>0$ in the above expression. For the second situation, $V_{W}=0$, after few manipulations, one gets

$$
D=\frac{C_{r}\left(V_{o}-V_{C_{r}}\left(t_{0}\right)\right) \cos \left(\omega_{r} t_{2}\right)}{C_{r}+C_{S}},
$$

which is also necessarily positive.

In a straight way, there exists ZVT in stage (iii) if and only if there exists a real positive root for (1). Due to the presence of the term $B t$, the analytical solution of $v(t)=0$ in (1) is not trivial. At this point, the circuit designer usually copes with the problem of checking occurrence of ZVT by means of numerical computation. However, this solution can be time consuming, depending on the size of step used in simulation, and may lead to an unreliable conclusion about the nonexistence of ZVT if the step is not adequately chosen. In this section, an approach that does not rely on numerical computation is given to decide rapidly and precisely if ZVT occurs or not in stage (iii).

First, from

$$
\frac{d v}{d t}=\dot{v}(t)=B+\omega_{e} C \cos \left(\omega_{e} t\right)-\omega_{e} D \sin \left(\omega_{e} t\right)=0,
$$

one can observe that (1) has stationary points (maxima or minima), which repeat with a period $2 \pi / \omega_{e}$. Suppose then $v\left(t^{*}\right)$ is the first minimum of $v(t)$ for $t>0$. The second minimum for $t>0$ is $v\left(t^{*}+2 \pi / \omega_{e}\right)$. From (1), it follows that $v\left(t^{*}+2 \pi / \omega_{e}\right)-v\left(t^{*}\right)=B 2 \pi / \omega_{e}$, which is always positive. Generalizing this reasoning, the next minimum always exceeds the previous by the amount $B 2 \pi / \omega_{e}$ and thus, the first minimum of $v(t)$ is the global minimum for $t>0$.

Since $v(t)$ is a continuous function and $v(0)>0$, there exists ZVT if and only if the first minimum of $v(t)$ for $t>0$, 
represented by $v\left(t^{*}\right)$, is less than or equal to zero. When $v\left(t^{*}\right)$ is equal to zero, one has the critical case for ZVT.

Hence, the main point of the proposed approach is to exchange the verification of occurrence of ZVT by means of zero cross detection or root calculation of (1) by the verification of the condition $v\left(t^{*}\right) \leq 0$. An analytical solution for this problem is given in next theorem.

Theorem 1: There exists ZVT in stage (iii) if and only if

$$
v\left(t^{*}\right)=A+B t^{*}+C \sin \left(\omega_{e} t^{*}\right)+D \cos \left(\omega_{e} t^{*}\right) \leq 0
$$

for

$$
t^{*}=\frac{1}{\omega_{e}}\left(\arcsin \left(\frac{-B}{\omega_{e} \sqrt{C^{2}+D^{2}}}\right)-\arctan \left(\frac{C}{-D}\right)\right) .
$$

Proof: From

$$
v(t)=A+B t+C \sin \left(\omega_{e} t\right)+D \cos \left(\omega_{e} t\right)
$$

one gets

$$
\begin{aligned}
& \dot{v}(t)=B+\omega_{e} C \cos \left(\omega_{e} t\right)-\omega_{e} D \sin \left(\omega_{e} t\right) \\
& =B+\omega_{e} \sqrt{C^{2}+D^{2}} \sin \left(\omega_{e} t+\arctan \left(\frac{C}{-D}\right)+2 k \pi\right)
\end{aligned}
$$

and

$$
\ddot{v}(t)=\omega_{e}^{2} \sqrt{C^{2}+D^{2}} \cos \left(\omega_{e} t+\arctan \left(\frac{C}{-D}\right)+2 k \pi\right)
$$

with $k$ integer. From the necessary condition for a minimum, given by $\dot{v}(t)=0$, one has

$$
\sin \left(\omega_{e} t+\arctan \left(\frac{C}{-D}\right)+2 k \pi\right)=\frac{-B}{\omega_{e} \sqrt{C^{2}+D^{2}}} .
$$

After few manipulations using the previous expressions for $B, C, D$ and $\omega_{e}$, it follows that

$$
\frac{-B}{\omega_{e} \sqrt{C^{2}+D^{2}}}=\frac{-I}{\sqrt{I^{2}+\omega_{e}^{2} C_{r}^{2}\left(V_{o}-V_{W}-V_{C_{r}}\left(t_{2}\right)\right)^{2}}} .
$$

Thus,

$$
\left.\sin \left(\omega_{e} t+\arctan \left(\frac{C}{-D}\right)+2 k \pi\right) \in\right]-1,0[
$$

and

$$
\left.\left(\omega_{e} t+\arctan \left(\frac{C}{-D}\right)+2 k \pi\right) \in\right] 3 \pi / 2,2 \pi[.
$$

This allows to conclude that $\ddot{v}(t)>0$ and then (21) is also sufficient to provide the minimum points of $v(t)$. Since

$$
\left.\arctan \left(\frac{C}{-D}\right) \in\right] \pi, 3 \pi / 2[
$$

due to the fact that $C<0$ and $D>0$, one has that the first minimum of $v(t)$ for $t>0$ occurs for $k=0$ in (21). Thus, the solution of (21) for $k=0$, given by (17) in Theorem 1 , is necessary and sufficient to provide the time of occurrence of the first minimum of $v(t)$ and (16) in Theorem 1 checks if this minimum is negative or null. This proves that Theorem 1 is necessary and sufficient to detect ZVT in stage (iii).

To illustrate the efficiency of Theorem 1 as a test to decide on the existence of ZVT, consider the circuit under study here with parameters given in Table I. Parameters $V_{W}$ and $V_{C r}\left(t_{0}\right)$ are given by the topology choice. In this case, the parameters used are from [8]. These parameters allow to obtain

$$
\omega_{e}=12.91 \times 10^{6} \mathrm{rad} / \mathrm{s}
$$

and

and then

$$
V_{C r}\left(t_{2}\right)=-611.0101 \mathrm{~V}
$$

$$
\begin{gathered}
A=-58.2576 V ; \\
B=2.5000 \times 10^{9} \mathrm{~V} / \mathrm{s} ; \\
C=-193.6492 \mathrm{~V} ;
\end{gathered}
$$

and

$$
D=458.2576 \mathrm{~V}
$$

Applying Theorem 1, one has that the first minimum for $t>0$ occurs at

and its value is

$$
t^{*}=181.41 n s
$$

$$
v\left(t^{*}\right)=-62.9967 \mathrm{~V}
$$

which allows to conclude rapidly and precisely, without computer simulation, that there exists ZVT for stage (iii) for the circuit with parameters in Table I. Figure 3 presents function $v(t)$ for this example and corroborates, based on numerical computation, the existence of ZVT. Observe that the choice of a time step not sufficiently small could lead to the wrong conclusion of nonexistence of ZVT in this case. The reliability of the conclusions based on numerical computation becomes computationally more expensive when ZVT approaches of the critical case, where $v(t)$ only touches the horizontal axis, without crossing it. In this case, the time step must be very sharp, increasing the computational time necessary to have a reliable conclusion. On the other hand, Theorem 1 copes with the decision of existence of ZVT without such problems of numerical precision, rapidly providing a response for the question.

Suppose that the circuit designer has a set of possible values for $L_{r}$ and $C_{r}$ for the auxiliary branch and must investigate for which pairs $\left(L_{r}, C_{r}\right)$ ZVT is ensured. As an example, suppose the circuit with parameters in Table I, except for $L_{r}$ and $C_{r}$, that now are given by $C_{r} \in[1,2, \ldots, 30] n F$ and $L_{r} \in[2,3, \ldots, 25] \mu H$.

These sets can be arbitrarily chosen by the circuit designer based on cost, precision and availability of the components, volume or other constraints. The prior sets provide a space of design with 720 points $\left(L_{r}, C_{r}\right)$. The investigation of ZVT for all the elements of this set by means of numerical computation can be time consuming. On the other hand, for each one of these pairs $\left(L_{r}, C_{r}\right)$, Theorem 1 can be applied to decide rapidly on the existence of ZVT. Using Matlab running in a notebook with Intel Core Duo processor, 1024 GB RAM, the test of all 720 pairs with Theorem 1 is carried out in $32 \mathrm{~ms}$, which is a considerably short time, providing the result shown in Figure 4. In this figure, the area shown in grey corresponds to the pairs $\left(L_{r}, C_{r}\right)$ for which ZVT operation is assured. Moreover, the method proposed has the

TABLE I Circuit parameters

\begin{tabular}{cc}
\hline Parameter & Value \\
\hline$V_{o}$ & $400 \mathrm{~V}$ \\
\hline$I$ & $10 \mathrm{~A}$ \\
\hline$V_{W}$ & $V_{o}$ \\
\hline$V_{C r}\left(t_{0}\right)$ & $-800 \mathrm{~V}$ \\
\hline$C_{S}$ & $1 n F$ \\
\hline$C_{r}$ & $3 n F$ \\
\hline$L_{r}$ & $8 \mu \mathrm{H}$
\end{tabular}




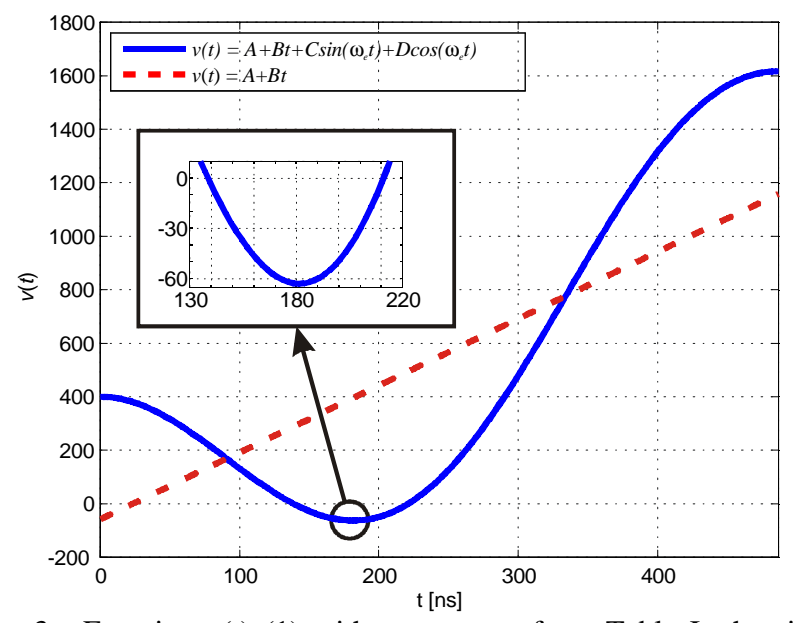

Fig. 3. Function $v(t)$ (1) with parameters from Table I, showing ZVT. The minimum of (1) is $-62.9967 \mathrm{~V}$ and occurs for $181.41 \mathrm{~ns}$.

advantage of being conclusive, while the numerical ones depend strongly on the initial guess and on the step (or error) previously specified.

\section{PROPOSED CONDITIONS}

The conditions proposed in the sequence allow finding a root for the nonlinear function (1). This equation is obtained by applying the Kirchhoff's Laws to the circuit formed by the series connection of capacitors $C_{S 1}$ (in parallel with current source $I$ ) and $C_{r}$ and inductor $L_{r}$.

In (1) the voltage $V_{C r}\left(t_{0}\right)$ is given by the topology under analysis. Some examples for chosen topologies are given in Table II.

By finding the root of (1), it can be determined the time of occurrence of ZVT and, as a consequence, the duration of stage (iii-a), which is useful for several analyses, as will be illustrated later on.

It is easy to notice that, by knowing the numerical values of $A, B, C, D$ and $\omega_{e}$ for a specific case, the root can be found using numerical methods [14]. However, the problem to be solved here is to find analytical expressions depending on $A$, $B, C, D$ and $\omega_{e}$ in order to provide a good approximation of

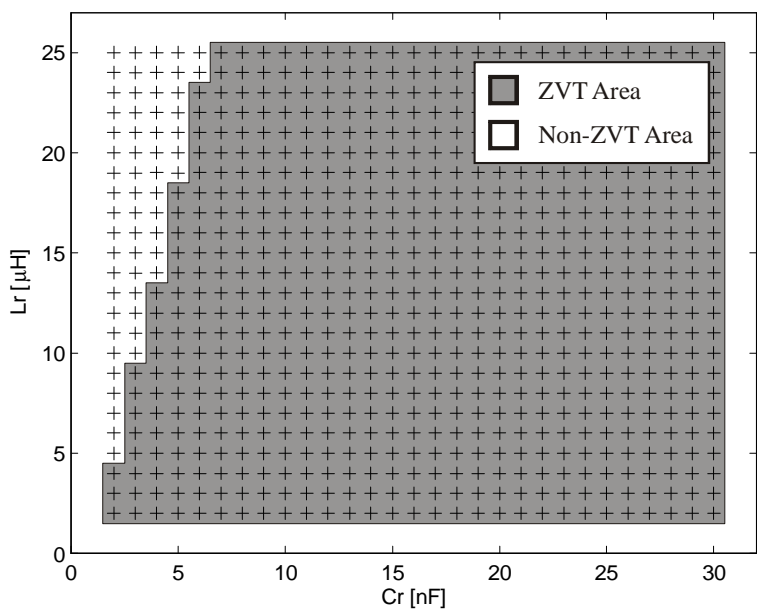

Fig. 4. Pairs $(\mathrm{Lr}, \mathrm{Cr})$ for which ZVT is ensured by Theorem 1. ZVT holds for 638 points of the set of 720 points tested.
TABLE II

Circuit parameters

\begin{tabular}{cccc}
\hline Topology & $\boldsymbol{V}_{\boldsymbol{C r}}\left(\boldsymbol{t}_{\mathbf{0}}\right)$ & $\boldsymbol{V}_{\boldsymbol{W}}$ & $\boldsymbol{V}_{\boldsymbol{C l a m p}}$ \\
\hline$[7],[10]$ & 0 & 0 & - \\
\hline$[8]$ & $-2 V_{o}$ & $V_{o}$ & 0 \\
\hline$[9]$ & undefined & 0 & - \\
\hline$[11]$ & $-V_{o}$ & 0 & $V_{o}$ \\
\hline
\end{tabular}

the root of (1). This is a more general solution for the problem and allows the use of the obtained expressions for analysis and design.

Section III of this paper provided a condition to determine if the root of (1) exists or not. Since $v(t)$ in (1) is continuous, with periodical extremum points and is necessarily positive for $t=0$, the root exists if and only if there is a negative minimum (or zero, in the critical case) for function (1). The time of occurrence of this minimum is given by $t^{*}$ in equation (17), referred as $t_{1 n}$ from now on, and is used as a starting point for the methods that follow, which are also a contribution of this paper.

Three approaches are given in this section to compute an approximation of the root of (1). Method 1 is based on a second order Taylor series approximation of $v(t)$ with the center of the series placed at $P_{\text {Root }}$ in Figure 5. Method 2 is based on a quadratic interpolation of (1) using points $P_{1}$ and $P_{2}$ and the time-derivative of $v(t)$ of the curve at $P_{1}$, as shown in Figures 6 and 7. Method 3 is similar to method 2 with the difference that is used the time-derivative at $P_{2}$ instead of $P_{l}$.

\section{A. Method 1}

The key idea here is to center a Taylor series approximation of $v(t)$ at $P_{\text {Root }}$. $P_{\text {Root }}$ is the root of the line connecting points $P_{1}$ and $P_{2}$, as seen in Figure 5, and is located at

$$
t_{P_{\text {Root }}}=t_{1 n} v(0) /\left(v(0)-v\left(t_{1 n}\right)\right) .
$$

The second order Taylor series of (1), centered at $t_{\text {PRoot }}$, is given by

Equivalently,

$$
\begin{aligned}
& v_{\text {Taylor }}(t)=v\left(t_{P_{\text {Root }}}\right)+\dot{v}\left(t_{P_{\text {Root }}}\right)\left(t-t_{P_{\text {Root }}}\right) . \\
& +\ddot{v}\left(t_{P_{\text {Root }}}\right)\left(t-t_{P_{\text {Root }}}\right)^{2} / 2
\end{aligned}
$$

$$
\begin{aligned}
& v_{\text {Taylor }}(t)=A+B t \\
& +C\left(\begin{array}{l}
\sin \left(\omega_{e} t_{P_{\text {Root }}}\right)+\omega_{e} \cos \left(\omega_{e} t_{P_{\text {Root }}}\right)\left(t-t_{P_{\text {Root }}}\right) \\
-0.5 \omega_{e}^{2} \sin \left(\omega_{e} t_{P_{\text {Root }}}\right)\left(t-t_{P_{\text {Root }}}\right)^{2}
\end{array}\right) . \\
& +D\left(\begin{array}{l}
\cos \left(\omega_{e} t_{P_{\text {Root }}}\right)-\omega_{e} \sin \left(\omega_{e} t_{P_{\text {Root }}}\right)\left(t-t_{P_{\text {Root }}}\right) \\
-0.5 \omega_{e}^{2} \cos \left(\omega_{e} t_{P_{\text {Root }}}\right)\left(t-t_{P_{\text {Root }}}\right)^{2}
\end{array}\right)
\end{aligned}
$$

Thus, the root of $v_{\text {Taylor }}(t)$, named now on as $\Delta_{t 3 a}$, immediately calculated by means of the well known Baskara's formula, is an approximation of the root of (1). The error associated with a second order Taylor series approximation of $v(t)$ can be written as [14]

$$
e_{\text {Taylor }}=\left|\frac{\dddot{v}(\zeta)}{3 !}\left(t-t_{P_{\text {Root }}}\right)^{3}\right|
$$

for some $\zeta$ in the open interval with extrema $t_{P R o o t}$ and $t$. From Figure 5, the actual root is located in the interval with extrema $t_{P R o o t}$ and $\hat{t}$, where $\hat{t}$ is the point where the derivative of $v(t)$ at $t_{\text {PRoot }}$ crosses the horizontal axis, being 
given by

$$
\hat{t}=t_{P_{\text {root }}}-\frac{v\left(t_{P_{\text {root }}}\right)}{\dot{v}\left(t_{P_{\text {root }}}\right)} \text {. }
$$

Hence, the error in the approximation in Method 1 can be bounded by

$$
e_{\text {Taylor }}<\frac{\omega_{e}^{3}\left(\sqrt{C^{2}+D^{2}}\right)}{6}\left|\left(\hat{t}-t_{P_{\text {root }}}\right)^{3}\right| .
$$

Although the results provided by this method are not very satisfactory, this method is shown because it is quite simple.

Two alternative methods are presented now.

\section{B. Method 2}

This method lies in interpolating two points of the $v(t)$ curve with a second order equation, given as below:

$$
v_{\text {int }}(t)=a t^{2}+b t+c,
$$

whose derivative is

$$
\overrightarrow{v_{\text {int }}} \dot{(}(t)=2 a t+b
$$

The points chosen to be part of (40) are the point of the first positive minimum and the point of half of the time of the first positive minimum of the real curve. This is done because the commutation occurs during this interval for the most critical cases. Then it is defined:

and

$$
P_{1}=\left[t_{1 n} / 2, \quad v\left(t_{1 n} / 2\right)\right]
$$

$$
P_{2}=\left[t_{1 n}, \quad v\left(t_{1 n}\right)\right] .
$$

Given that only two points do not provide enough information to define a second order interpolating equation, there must be a third constraint. Such constraint is chosen to be the derivative of the curve in one of the points that are being interpolated, Figure 6 . In this way, two points can be analyzed.

Initially the third constraint analyzed is the derivative of curve $v(t)$ at $P_{1}$ :

Then, it is defined

$$
\dot{P}_{1}=\left[t_{1 n} / 2, \quad v\left(t_{1 n} / 2\right)\right],
$$

where the derivative of $v(t)$ is given by

$$
\dot{v(t)}=B+\sqrt{C^{2}+D^{2}} \cos \left(\omega_{e} t+\arctan 2(D, C)\right) \omega_{e}
$$

and $\arctan 2()$ is the four quadrant inverse tangent function.

Points $P_{1}$ and $P_{2}$ must verify (40), resulting in the first and second lines of equation (46), respectively. Point $\dot{P}_{1}$ must

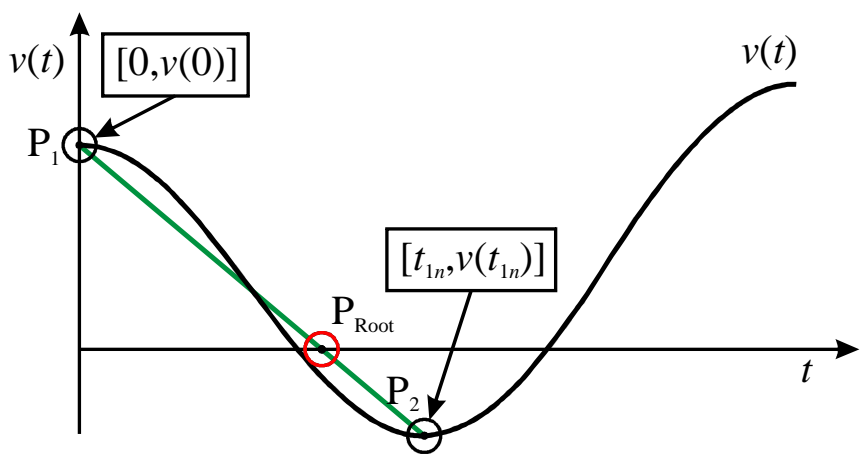

Fig. 5. Concept of method 1.

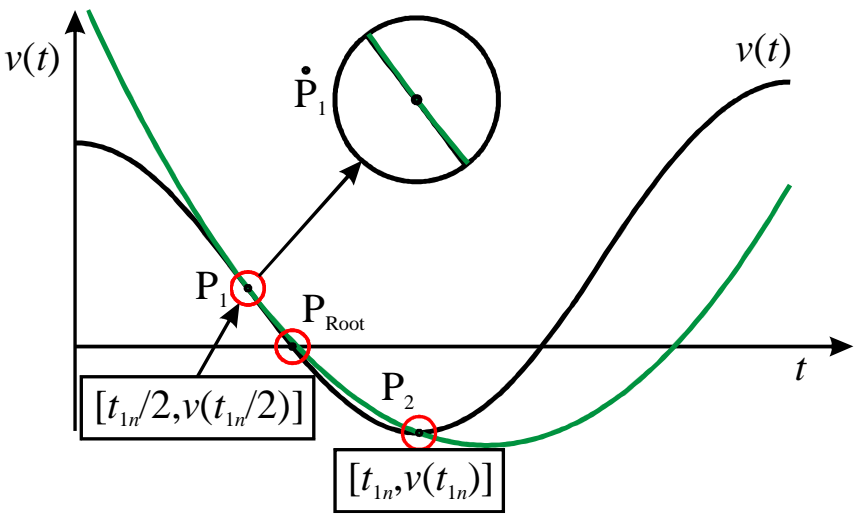

Fig. 6. Concept of method 2.

verify $v_{\text {int }}(t)$, given in (41), corresponding to the third line in (46). Thus, the following linear system is obtained:

$$
\left[\begin{array}{ccc}
\left(t_{1 n} / 2\right)^{2} & t_{1 n} / 2 & 1 \\
t_{1 n}{ }^{2} & t_{1 n} & 1 \\
2 t_{1 n} / 2 & 1 & 0
\end{array}\right]\left[\begin{array}{l}
a \\
b \\
c
\end{array}\right]=\left[\begin{array}{c}
v\left(t_{1 n} / 2\right) \\
v\left(t_{1 n}\right) \\
v\left(t_{1 n} / 2\right)
\end{array}\right] .
$$

By solving (46), the parameters $a, b$ and $c$ can be determined, resulting in a second order curve that interpolates $P_{1}$ and $P_{2}$ and has a derivative in point $P_{1}$ given by $\dot{P}_{1}$.

With $v_{\text {int }}(t)$ determined, its root $\left(t_{\text {PRoot }}\right)$ can be calculated easily by using Baskara's formula and, in this way, the Taylor series is centered at $t_{P R o o t}$.

\section{Method 3}

Another constraint that can be utilized is the derivative of the curve $v(t)$ on $P_{2}$, Figure 7:

Then, it is defined

$$
\dot{P}_{2}=\left[t_{1 n}, \quad v\left(\dot{t_{1 n}}\right)\right],
$$

where the derivative of $v\left(t_{1 n}\right)$ is obtained by making $t=t_{1 n}$ in (45).

Lines 1 and 2 in (48) are as those in (46). The third line is obtained by applying $P_{2}$ in (41). Thus, the following linear system is obtained:

$$
\left[\begin{array}{ccc}
\left(t_{1 n} / 2\right)^{2} & t_{1 n} / 2 & 1 \\
t_{1 n}{ }^{2} & t_{1 n} & 1 \\
2 t_{1 n} & 1 & 0
\end{array}\right]\left[\begin{array}{l}
a \\
b \\
c
\end{array}\right]=\left[\begin{array}{c}
v\left(t_{1 n} / 2\right) \\
v\left(t_{1 n}\right) \\
v\left(\dot{t_{1 n}}\right)
\end{array}\right] .
$$

By solving (48), the parameters $a, b$ and $c$ can be determined, resulting in a second order curve that interpolates $P_{1}$ and $P_{2}$ and has a derivative in point $P_{2}$ given by $\dot{P}_{2}$.

Then, $t_{P R o o t}$ can be calculated from $v_{\text {int }}(t)$, as it was done before, and the Taylor series can be centered on this point.

\section{RESULTS}

The efficiency of the proposed methods to compute good approximations of the root of (1) is illustrated by means of the following numerical examples. 


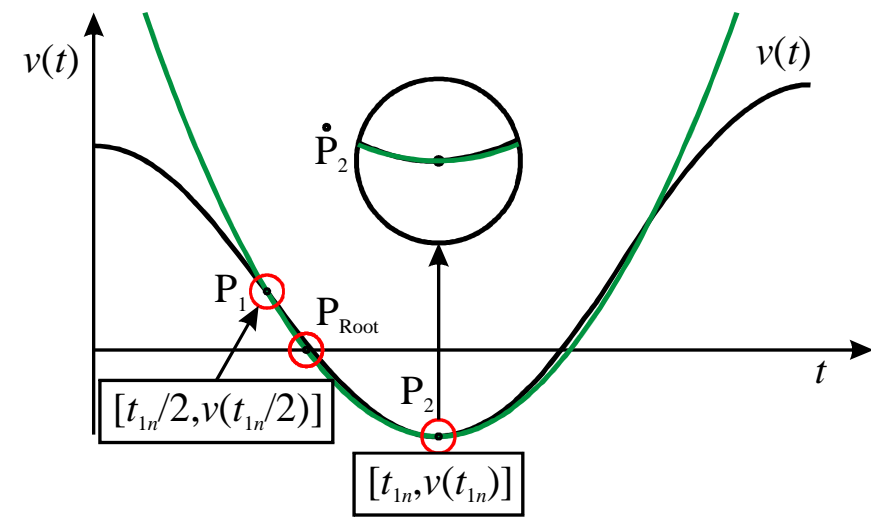

Fig. 7. Concept of method 3.

1) Example for Method 1:

Consider the parameters in (1) given in Table I.

Figure 8 shows function $v(t)$ for the above parameters; the second order Taylor series approximation of $v(t)$ is centered at $P_{\text {Root }}$. It is important to observe from this figure that the second order Taylor series used to approximate $v(t)$ provides a good approximation around the center $P_{\text {Root }}$, which includes the region of the actual root, as pointed out in the figure. The parts of $v_{\text {Taylor }}$ that are not good approximations of $v(t)$ are irrelevant for the computation of the root of $v(t)$. The approximation of the root of $v(t)$, computed by Method 1, is given by $138.81 \mathrm{~ns}$, and the actual root, numerically computed [13], is given by $139.40 n s$. This illustrates a very good approximation of the actual root, provided by Method 1 in this example.

The upper bound of the error, given by Method 1, is $2.3788 \mathrm{~V}$. The actual error, that is, the modulus of $v(138.81)$ is given by $0.72 \mathrm{~V}$, thus respecting the upper bound provided by Method 1.

2) Example for Method 2:

Using the same parameters from the former example the system obtained is

$$
\left[\begin{array}{ccc}
8.22 \times 10^{-15} & 9.07 \times 10^{-8} & 1 \\
3.29 \times 10^{-14} & 1.81 \times 10^{-7} & 1 \\
1.81 \times 10^{-7} & 1 & 0
\end{array}\right]\left[\begin{array}{l}
a \\
b \\
c
\end{array}\right]=\left[\begin{array}{c}
168.50 \\
-63.00 \\
-3.92 \times 10^{9}
\end{array}\right] .
$$

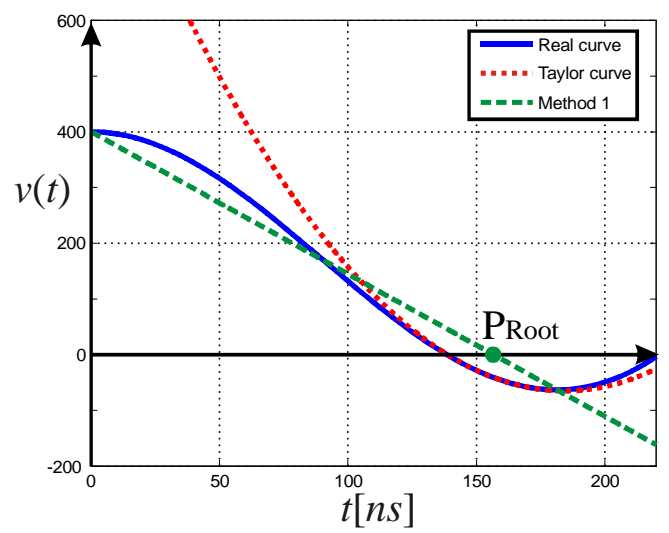

Fig. 8. Function $v(t)$, second order Taylor series approximation of $v(t)$, marked as vTaylor. The Taylor series is centered at $P_{\text {Root. }}$
From which, the first curve is obtained,

$$
v_{\text {int }}(t)=1.51 \times 10^{16} x^{2}-6.66 \times 10^{9} x+648.59 \text {. }
$$

The first positive root for $v_{\text {int }}(t)$ is at $145.03 n s$. The root of $V_{\text {taylor }}(t)$ centered at this point is $138.56 n s$, while this value calculated numerically is $139.40 \mathrm{~ns}$. This shows that the root is calculated with great precision. This method is illustrated in Figure 9.

3) Example for Method 3:

Using the same parameters from the former example the following system is obtained:

$$
\left[\begin{array}{lcl}
8.22 \times 10^{-15} & 9.07 \times 10^{-8} & 1 \\
3.29 \times 10^{-14} & 1.81 \times 10^{-7} & 1 \\
3.62 \times 10^{-7} & 1 & 0
\end{array}\right]\left[\begin{array}{l}
a \\
b \\
c
\end{array}\right]=\left[\begin{array}{c}
168.50 \\
-63.00 \\
0
\end{array}\right],
$$

from which the following curve it is obtained,

$$
v_{\text {int }}(t)=2.81 \times 10^{16} x^{2}-1.02 \times 10^{10} x+863 \text {. }
$$

The first positive root for $v_{\text {int }}(t)$ is at $134.09 n s$. The root of $V_{\text {taylor }}(t)$ centered at this point is $138.54 n s$, while this value calculated numerically is $139.40 \mathrm{~ns}$. Again, the root was calculated with great precision. This method is illustrated in Figure 10.

As a final example for validation of the proposed conditions, consider the converter topology in [8] with parameters given in Table I. The simulation results for this converter can be seen in Figure 11, indicating that the ZVT time obtained is about $138 \mathrm{~ns}$. All proposed methods for determination of the ZVT time yielded values within the interval [138, 139]ns. Thus, there exists a good correspondence between the theoretical proposal and the simulated results. Besides, the VS1 waveform clearly indicates the occurrence of ZVT. This fact can also be observed in Figure 4, since the pair $\left(L_{r}, C_{r}\right)$ utilized belongs to the ZVT area provided by Theorem 1 .

By means of the approaches proposed herein some important design parameters can be obtained and evaluated, such as the operation sequence, the total commutation time, the RMS values of current through the auxiliary circuit, resistive losses through the auxiliary components, loss of duty ratio, to name a few. Below two of these parameters are given as examples.

A. Operation Mode:

The operation mode can be determined by evaluating the following expression

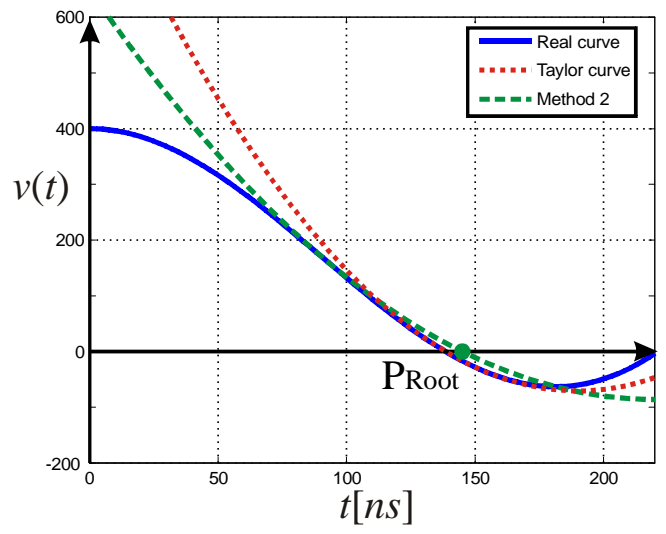

Fig. 9. Example for method 2. 
where

$$
v_{C r}\left(\Delta_{t 3 a}\right)>V_{\text {Clamp }}
$$

$$
v_{C r}(t)=A-V_{W}+B t-\left(C \sin \left(\omega_{e} t\right)+D \cos \left(\omega_{e} t\right)\right) \frac{C_{s}}{C_{r}} .
$$

$\Delta_{t 3 a}$ is obtained from the methods shown in the former section.

When (53) is satisfied, the converter operates in sequence 2, i.e., $C_{r}$ clamps before the voltage of $C_{S}$ becomes null. Else it may operate either in sequence 1 or in sequence 3 .

It operates in sequence 3 if $C_{r}$ is clamped after the $C_{S}$ voltage becomes null, satisfying (55).

$$
\sqrt{\left(V_{C_{r}}\left(\Delta_{t 3 a}\right)+V_{W}\right)^{2}+\left(I_{L_{r}}\left(\Delta_{t 3 a}\right) Z_{r}\right)^{2}} \geq V_{\text {Clamp }},
$$

where $V_{C r}\left(\Delta_{t 3 a}\right)$ is obtained by making $t=\Delta_{t 3 a}$ in (54) and

$$
\begin{aligned}
& I_{L r}\left(\Delta_{t 3 a}\right)=\left(-C \cos \left(\omega_{\mathrm{e}} \Delta_{t 2}\right)+D \sin \left(\omega_{\mathrm{e}} \Delta_{t 2}\right)\right) C_{S} \omega_{\mathrm{e}} ; \\
& +B C_{r}
\end{aligned}
$$

$$
\begin{gathered}
\Delta_{t 2}=\arcsin \left(\frac{-I Z_{r}}{V_{C r}\left(t_{0}\right)+V_{W}-V_{o}}\right) / \omega_{r} ; \\
\omega_{r}=\sqrt{1 / L_{r} C_{r}} .
\end{gathered}
$$

$Z_{r}$ is given in (11) and $V_{C r}\left(t_{0}\right)$ is defined by the topology, according to Table I.

Otherwise, the converter operates in sequence 1 .

\section{B. Commutation Time}

The commutation time is determined depending on the operation sequence of the converter.

\section{1) Sequence 1}

The amount of time used to accomplish the transitions by the converter when operating in this sequence is given by:

$$
t_{\text {Com }}=\Delta_{t 1}+\Delta_{t 2}+\Delta_{t 3 a}+\Delta_{t 4 a}+\Delta_{t 5}+\Delta_{t 6}+\Delta_{t 7} \text {, }
$$

where $\Delta_{t 1}$ and $\Delta_{t 6}$ are given by the modulation scheme utilized; $\Delta_{t 2}$ is given by (57); $\Delta_{t 3 a}$ can be determined by the methods presented in section III;

$$
\Delta_{t 4 a}=-\arctan \left(\frac{V_{C r}\left(\Delta_{t 3 a}\right)+V_{W}}{I_{L r}\left(\Delta_{t 3 a}\right) Z_{r}}\right) / \omega_{r},
$$

where $V_{C r}\left(t_{3 a}\right)$ is obtained by making $t=\Delta_{t 3 a}$ in (54) and

$$
\begin{gathered}
I_{L r}\left(\Delta_{t 3 a}\right)=\left(D \sin \left(\omega_{\mathrm{e}} \Delta_{t 2}\right)-C \cos \left(\omega_{\mathrm{e}} \Delta_{t 2}\right)\right) C_{S} \omega_{\mathrm{e}}+B C_{r} \\
\Delta_{t 5}=\pi / \omega_{r}
\end{gathered}
$$

and

$$
\Delta_{t 7}=\left(V_{o} / I\right) C_{S}
$$

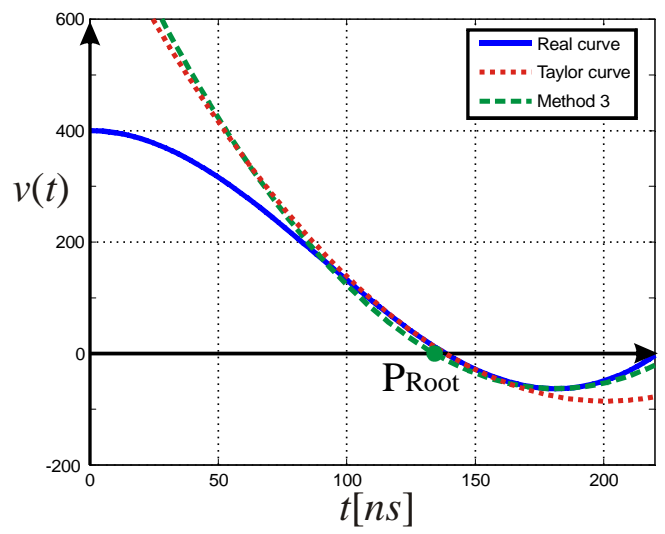

Fig. 10. Example for method 3.

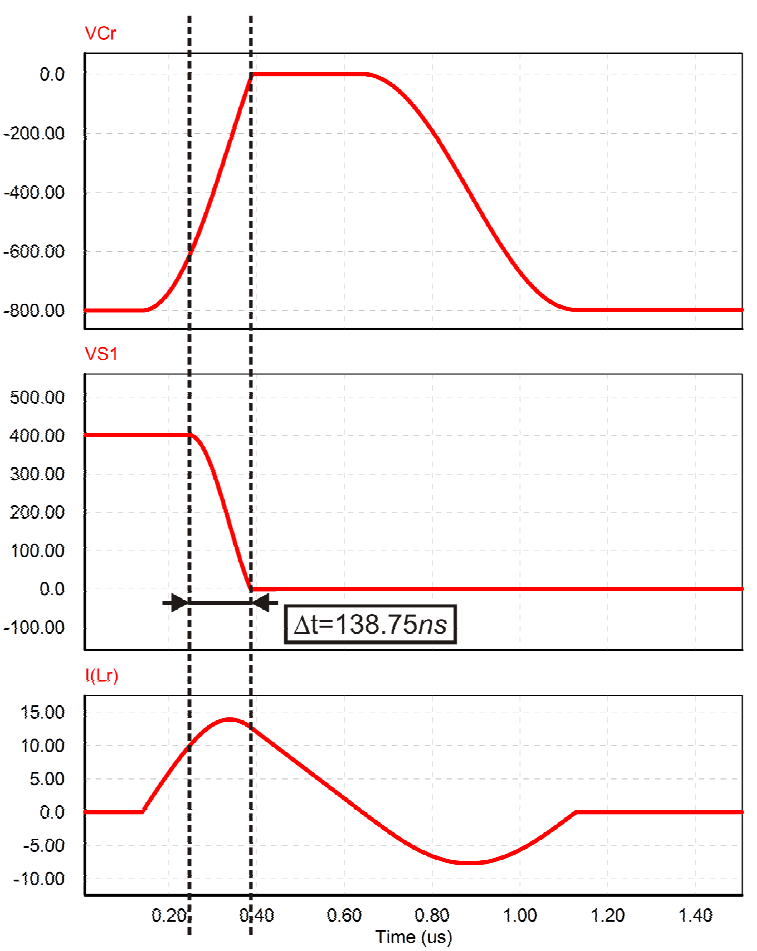

Fig. 11. Simulation results for converter topology [8] with the parameters from Table I.

2) Sequence 2:

The amount of time used to accomplish the transitions by the converter when operating in this sequence is given by:

$$
\begin{aligned}
& t_{C o m}=\Delta_{t 1}+\Delta_{t 2}+\Delta_{t 3 a}+\Delta_{t 3 b}+\Delta_{t 4 b}, \\
& +\Delta_{t 5}+\Delta_{t 6}+\Delta_{t 7}
\end{aligned}
$$

where $\Delta_{t 1}$ and $\Delta_{t 6}$ are given by the modulation scheme utilized; $\Delta_{t 2}$ is given by (57); $\Delta_{t 3 a}$ can be obtained by the approaches presented in section III, making $v_{\text {Taylor }}(t)=V_{\text {Clamp }}$ and solving for $t=\Delta_{t 3 a}$.

$$
\begin{aligned}
& \Delta_{t 3 b}=\frac{-\pi}{\omega_{s}} \\
& -\frac{\pi-\arctan 2\left(V_{C s}\left(\Delta_{t 3 a}\right)-V_{o},\left(I-I_{L r}\left(\Delta_{t 3 a}\right)\right) Z_{s}\right)}{\omega_{s}} \\
& -\frac{\arcsin \left(\frac{V_{o}}{\sqrt{\left(V_{C s}\left(\Delta_{t 3 a}\right)-V_{o}\right)^{2}+\left(\left(I-I_{L r}\left(\Delta_{t 3 a}\right)\right) Z_{s}\right)^{2}}}\right)}{\omega_{s}}
\end{aligned}
$$

where $V_{C s}\left(\Delta_{t 3 a}\right)$ is obtained by making $t=\Delta_{t 3 a}$ in (1) and $I_{L r}\left(\Delta_{t 3 a}\right)$ is given in (61).

$$
\Delta_{t 4 b}=\frac{I_{L r}\left(\Delta_{t 3 b}\right)}{V_{\text {Clamp }}+V_{W}} L_{r},
$$

where

$$
\begin{aligned}
& I_{L r}\left(\Delta_{t 3 b}\right)=I_{L r}\left(\Delta_{\mathrm{t} 3 a}\right) \cos \left(\omega_{\mathrm{r}} \Delta_{t 3 b}\right) \\
& -\frac{V_{C r}\left(\Delta_{\mathrm{t} 3 a}\right)+V_{W}}{Z_{r}} \sin \left(\omega_{\mathrm{r}} \Delta_{t 3 b}\right)
\end{aligned} ;
$$

$\Delta_{t 5}$ and $\Delta_{t 7}$ are given by (62) and (63), respectively. 
3) Sequence 3:

The amount of time used to accomplish the transitions by the converter when operating in this sequence is given by:

$$
\begin{aligned}
& t_{\text {Com }}=\Delta_{t 1}+\Delta_{t 2}+\Delta_{t 3 a}+\Delta_{t 4 a}, \\
& +\Delta_{t 4 b}+\Delta_{t 5}+\Delta_{t 6}+\Delta_{t 7}
\end{aligned}
$$

where $\Delta_{t 1}$ and $\Delta_{t 6}$ are given by the modulation scheme utilized; $\Delta_{t 2}$ is given by (57); $\Delta_{t 3 a}$ can be obtained by the approaches presented in section IV;

$$
\begin{aligned}
& \Delta_{t 4 a}=\frac{\arcsin \left(\frac{V_{W}+V_{C l a m p}}{\sqrt{\left(V_{C r}\left(\Delta_{t 3 a}\right)+V_{W}\right)^{2}+\left(I_{L r}\left(\Delta_{t 3 a}\right) Z_{r}\right)^{2}}}\right)}{-\arctan 2\left(V_{C r}\left(\Delta_{t 3 a}\right)+V_{W}, I_{L r}\left(\Delta_{t 3 a}\right) Z_{r}\right) / \omega_{r}}
\end{aligned}
$$

where $V_{C r}\left(\Delta_{t 3 a}\right)$ is obtained by making $t=\Delta_{t 3 a}$ in (54) and $I_{L r}\left(\Delta_{t 3 a}\right)$ is given in (61).

$$
\Delta_{t 4 b}=\frac{I_{L r}\left(\Delta_{t 3 b}\right)}{V_{\text {Clamp }}+V_{W}} L_{r},
$$

where

$$
\begin{aligned}
& I_{L r}\left(\Delta_{t 3 b}\right)=I_{L r}\left(\Delta_{\mathrm{t} 3 a}\right) \cos \left(\omega_{\mathrm{r}} \Delta_{t 3 b}\right) \\
& -\frac{V_{C r}\left(\Delta_{\mathrm{t} 3 a}\right)+V_{W}}{Z_{r}} \sin \left(\omega_{\mathrm{r}} \Delta_{t 3 b}\right)
\end{aligned} ;
$$

$\Delta_{t 5}$ and $\Delta_{t 7}$ are given by (62) and (63), respectively.

\section{CONCLUSION}

This work proposed an analysis tool to verify the softswitching conditions for a given set of components $\left(L_{r}, C_{r}\right)$ for ZVT converters with resonant voltage source.

In addition, three numerical methods to compute the time of occurrence of ZVT for power converters with capacitor on the auxiliary resonant branch were presented.

The conditions in the paper provide analytical expressions to compute the time of occurrence of ZVT based on the converter parameters. The results obtained become useful for analysis and design, allowing evaluating operation sequence, total commutation time, resistive losses and other important parameters to describe the converter performance.

Examples provided in the text illustrate the efficiency of the proposed conditions.

\section{ACKNOWLEDGEMENT}

The authors would like to express their gratitude to "Coordenação de Aperfeiçoamento de Pessoal de Nível Superior - CAPES" and "Conselho Nacional de Desenvolvimento Científico e Tecnológico - CNPQ" (procs. $n^{\circ} 307232 / 2009-3$ and 478154/2009-7) for financial support.

\section{REFERENCES}

[1] P. Das, G. Moschopoulos, "A comparative study of zero-current-transition PWM converters", Trans. on Industrial Electronics, vol. 54, no. 3, pp. 1319-1328, June 2007.

[2] L. Schuch, J.R. Pinheiro, "Novo Princípio de Integração de Circuitos de Auxílio à Comutação: O Estudo do caso ZVT", Eletrônica de Potência, vol. 13, no. 1, Fevereiro de 2008, p. 1- 14;
[3] C.-M. Wang, "Novel zero-voltage-transition PWM DCDC converters", Trans. on Industrial Electronics, vol. 53, no. 1, pp. 254- 262, Feb 2006.

[4] M. Mezaroba, J.S. Scholtz, P.S.G. Giacomini, "Conversor CC-CC Elevador/Abaixador ZVS PWM com Grampeamento Ativo", Eletrônica de Potência, vol. 12, no. 3, Novembro de 2007, p. 197-204.

[5] M.L. Martins, J.L. Russi, J.R. Pinheiro, H.L. Hey, "Zero-Current Zero-Voltage Transition PWM Converters with Magnetically Coupled Auxiliary Circuit", Eletrônica de Potência, vol. 13, no. 4, Novembro de 2008, p. 201-208.

[6] M. Mezaroba, J.D. Sperb, "Conversor Auxiliar com Comutação ZVS aplicado ao Snubber de Undeland Regenerativo", Eletrônica de Potência, vol. 13, no. 2, Maio de 2008, p. 61-68.

[7] K.M. Smith and K.M. Smedley, "A comparison of voltage-mode softswitching methods for PWM converters," IEEE Trans. on Power Electronics, vol. 12, no. 2, pp. 376-386, Mar. 1997.

[8] L. Yang and C.Q. Lee, "Analysis and design of boost zero-voltage transition PWM converter," in Proc. IEEE APEC, pp. 707-713,1993.

[9] G. Moschopoulos, P. Jain, and G. Joos, "A novel zerovoltage switched PWM boost converter," in Proc. IEEE PESC, 1995, pp. 694-700.

[10]C.-J. Tseng and C.-L. Chen, "Novel ZVT-PWM converters with active snubbers," IEEE Trans. on Power Electronics, vol. 13, no. 5, pp. 861-869, Sep. 1998.

[11]D. M. Xu, J. M. Zhang, Y. C. Ren, and Z. Qian, “A novel single-phase active-clamped ZVT-PWM PFC converter," in Proc. IEEE APEC, 2000, pp. 456-459.

[12] M.L. Martins, J.L Russi, H.L. Hey, "Novel Design Methodology and Comparative Analysis for ZVT PWM Converters with Resonant Auxiliary Circuit", IEEE Trans. on Industry Applications, v. 42, n. 3, p. 779-796, 2006.

[13]P. Venkataraman, Applied Optimization with Matlab Programming, a Wiley-Interscience Publication, New York, USA, 2002.

[14]R.L. Burden, J.D. Faires, Numerical Analysis, $8^{\text {th }}$ edition, Ed. Thomson, Belmont, CA, USA, 2005.

\section{BIOGRAPHIES}

Vinícius Foletto Montagner was born in Faxinal do Soturno, RS, Brazil, in 1974. He received the B.S. and M.S. degrees Electrical Engineering from Federal University of Santa Maria in 1996 and 2000, respectively. He received the $\mathrm{PhD}$ and post-doctoral degrees in Electrical Engineering from University of Campinas in 2005 and 2006, respectively. $\mathrm{He}$ also spent a year in Berkeley University, CA, in his doctoral internship. Currently, he is a professor at Electric Power Processing Department in Federal University of Santa Maria. His research interests include control theory and its applications.

Hélio Leães Hey was born in Santa Maria, Brazil, in 1961. He received the B.S. degree from the Catholic University of Pelotas, Pelotas, Brazil, in 1985, and the M.S. and Ph.D. degrees from the Federal University of Santa 
Catarina, Florianópolis, Brazil, in 1987 and 1991, respectively. From 1989 to 1993, he was with the Federal University of Uberlândia, Uberlândia, Brazil. Since 1994, he has been with the Federal University of Santa Maria, where he is currently a Professor. He is also with the Power Electronics and Control Research Group (GEPOC). From 1995 to 1999, he was Editor of the Brazilian Power Electronics Journal. From 2005 to 2006, he was with the Electrical and Electronic Engineering Department, University of Oviedo, Oviedo, Spain, where he performed his postdoctoral research on power-factor-correction and soft-switching techniques. He has authored more than 140 papers published in power electronics journals and conference proceedings. His research interests include softswitching techniques, high-frequency power conversion, powerfactor-correction techniques, and renewable-energy applications.Group (GEPOC). From 1995 to 1999, he was Editor of the Brazilian Power Electronics Journal. From 2005 to 2006, he was with the Electrical and Electronic Engineering Department, University of Oviedo, Oviedo, Spain, where he performed his postdoctoral research on power-factor-correction and soft-switching techniques. He has authored more than 140 papers published in power electronics journals and conference proceedings. His research interests include soft-switching techniques, highfrequency power conversion, power factor-correction techniques, and renewable-energy applications.
Mário Lúcio da Silva Martins was born in Palmeira das Missões, RS, Brasil, in 1976. He received the B.S., M.S. and $\mathrm{Ph} . \mathrm{D}$. degrees in electrical engineering from the Federal University of Santa Maria, Santa Maria, Brazil, in 2000, 2002, and 2008, respectively. Since 2006, he has been with the Federal University of Technology - Paraná (UTFPR), Pato Branco, Brazil, where he is currently a Professor in Graduation and Pos-Graduation courses of Electrical Engineering, lecturing Electronics, Power Electronics and Circuit Analysis. His research interests include highperformance power converters, renewable-energy applications and variable speed drives. Dr. Martins was the recipient of the Second Prize Paper Award from the Industrial Power Converter Committee of the IEEE Industry Applications Society (IAS) at the 2005 IAS Annual Meeting and has authored 36 papers in power electronics journals and conference proceedings. Dr. Martins is a member of IEEE Power Electronics, Industrial Electronics and Industry Application Societies, and SOBRAEP.

Jumar Luís Russi was born in Passo Fundo, Brazil, in 1977. He received the B.S., M.S. and Ph.D. degrees from the Federal University of Santa Maria, Santa Maria, Brazil, in 2000, 2003 and 2007, respectively, in electrical engineering. Since 2007 he is a professor at the Federal University of Pampa in Alegrete-RS. His research interests include switched-mode power supplies, uninterruptible power supplies and high performance power converters. 